\title{
A simple ghost fluid method for compressible multicomponent flows with capillary effects
}

\author{
Nikolaos Bempedelis, Yiannis Ventikos* \\ Department of Mechanical Engineering, University College London, WClE 7JE, United Kingdom
}

\begin{abstract}
A novel way of implementing surface tension effects in sharp-interface compressible flow models is proposed, aiming to address problems where liquid compressibility and capillarity are both important. The method is built on the principles of the grid-aligned formulation for ghost fluid techniques. In this approach, the Riemann problems at the interface are formulated along the grid rather than in a normal-to-the-interface direction; the method is thus simpler. The performance of the method is thoroughly examined following implementation in a well-established front tracking framework.
\end{abstract}

Keywords: Multiphase flows, Compressible flows, Surface tension, Ghost fluid method, Front tracking

\section{Introduction}

Surface tension is a prominent characteristic of multiphase flows, related to phenomena such as the rise of fuel in oil lamps, the floating of water striders and the development of tears on a wine glass. Despite its (possibly strong) influence on the flow, implementation of surface tension effects in numerical methods has only recently reached a certain degree of maturity (see the review by Popinet [1]). In the majority of presently existing methods, the flow is modelled as incompressible. In flows where compressibility is important, the effects of surface tension are often neglected due to the associated high velocities, which imply that the flow is driven mainly by inertia. However, there are cases, particularly when considering small-scale flows, where both compressibility and capillarity are of importance; examples include the high-speed breakup or impact of a droplet and the interaction of a bubble with free surfaces, waves, or other bubbles. The number of methods that have been developed for such flows is rather limited (e.g. [2, 3, 4] for inviscid flows and [5, 6, 7, 8, 9, 10] for cases where viscous effects are also considered).

In the current work, we present a novel way of incorporating capillary action within a sharp-interface compressible flow framework (section 2). The method is based on the principles of the grid-aligned formulation for ghost fluid methods [11]. The behaviour of the model is examined through a series of challenging test cases (section 3). Finally, the method is applied to the study of a problem where compressibility and capillary effects are both important.

\section{Methodology}

Surface tension is a force that acts on interfaces; this creates the need to locate them and track their motion. The developed method is implemented within the front tracking framework ${ }^{1}[12,13,14,8]$, which provides highly accurate

*Corresponding author.

Email address: y.ventikos@ucl .ac.uk (Yiannis Ventikos)

${ }^{1}$ http://www.ams.sunysb.edu/ linli/FronTier++_Manual/index.html. 
tracking of interfaces and robust treatment of topology changes [15], but is compatible with other interface tracking methods as well (e.g. level sets, where topology changes are handled automatically).

The flows are modelled by the compressible Euler equations, together with an equation for the total energy. The system of equations is closed with the stiffened gas equation of state. The thermodynamic parameters for the fluids under consideration are taken from [11]. Surface tension forces are accounted for through appropriate jump conditions at the fluid interfaces (section 2.1).

The fluids are assumed immiscible, and the effects of diffusion (viscous and thermal) and phase change are neglected. The reason for omitting viscosity is the following: the lack of physical diffusion mechanisms represents a significant challenge for the numerical method, as any unphysical oscillations will not be damped. This is of particular importance from a method development point of view: implementation of capillary action prior to the consideration of viscous effects is critical for the rigorous assessment of the solver's behaviour, before viscosity masks unwanted effects. A $5^{\text {th }}$-order WENO scheme is used for the calculation of the fluxes. The equations are advanced in time using an RK3 method. An RK2 method is used for the material interface advection equation.

\subsection{The grid-aligned ghost fluid method with surface tension}

The ghost fluid method, originally introduced in [16], is a boundary condition capturing method for the treatment of interfaces and discontinuities. The different phases are essentially considered independently and are coupled via enforcement of jump conditions at the interfaces, in an attempt to prevent oscillations and maintain the discontinuities sharp. Together with a number of variants (e.g. $[17,18]$ ), ghost fluid methods have been widely used in computations of multiphase flows.

The principal idea of the grid-aligned ghost fluid method [11] is to express the jump conditions for the interface following its mapping to the underlying Eulerian grid (i.e. fully adopting the stair-stepped representation, figure 1b) rather than in a normal to the interface direction, as typically done in ghost fluid methods (figure 1a). This is an approach closely related to $[19,20]$. In this way, the coupling of the flows is considerably simplified (compared to standard multidimensional formulations), conferring benefits related to computational cost and facility of solver development/validation. At the same time, the method was shown to be capable of yielding results with accuracy that is close or equal to existing methods [11].

In the present work, we show that surface tension forces may be consistently accounted for within such a gridaligned framework. The concept of the proposed method is schematically depicted in figure 1c. The interface for which we express the interface conditions remains aligned to the grid, as in the original method [11], but is locally curved instead of being planar.

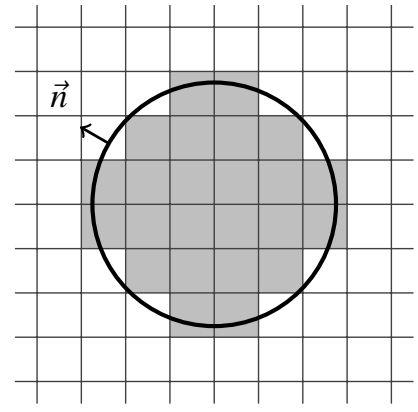

(a) Standard ghost fluid methods.

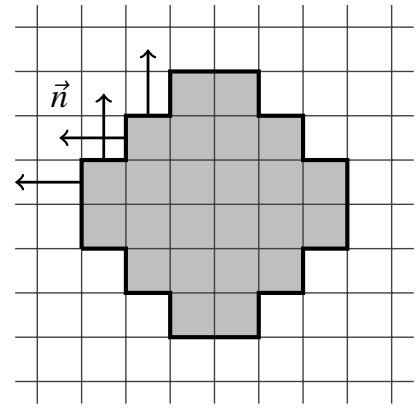

(b) Grid-aligned ghost fluid method.

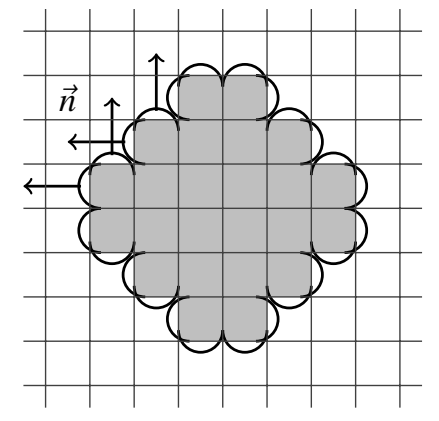

(c) Grid-aligned ghost fluid method with surface tension.

Figure 1: Schematic representation of different formulations for ghost fluid methods. Cells filled with different components are distinguished by shading. Gridlines represent cell edges. The interface as perceived for the computation of the ghost states is denoted with a thick black line. The interface curvature in (c) is exaggerated for illustrative purposes. 
In the grid-aligned ghost fluid method [11], the single-phase stencils are completed by enforcing Dirichlet-type boundary conditions at the interface. The states of these conditions are the states of the fluids at the interface. In the present work, these are computed using a modified version of the linearized interface interaction method [17], accounting for the pressure jump across the interface due to surface tension by enforcing the constraint $p_{I, l}-p_{I, r}=\sigma \kappa_{I} n$, where the subscript ()$_{I}$ denotes the interface, subscripts ()$_{l, r}$ denote its two sides, $\sigma$ is the surface tension coefficient and $\kappa_{I}=\left(\kappa_{l}+\kappa_{r}\right) / 2$ the curvature. In the adopted approach, the interface is aligned with the grid, and its normal may only take the values $\boldsymbol{n}= \pm(1,0,0), \pm(0,1,0)$ or $\pm(0,0,1)$. The scalar variable $n$ hence refers to the only non-zero component of the interface normal vector, $n= \pm 1$. As in [17], the states are computed using the method of characteristics, but different types of Riemann solvers may be considered (e.g. [18]). The interface states are,

$$
\begin{aligned}
& u_{I}=\frac{\rho_{l} c_{l} u_{l}+\rho_{r} c_{r} u_{r}+p_{l}-p_{r}}{\rho_{l} c_{l}+\rho_{r} c_{r}}-\frac{\sigma \kappa_{I} n}{\rho_{l} c_{l}+\rho_{r} c_{r}}, \\
& p_{I, l}=\frac{\rho_{l} c_{l} p_{r}+\rho_{r} c_{r} p_{l}+\rho_{l} c_{l} \rho_{r} c_{r}\left(u_{l}-u_{r}\right)}{\rho_{l} c_{l}+\rho_{r} c_{r}}+\frac{\sigma \kappa_{I} n \rho_{l} c_{l}}{\rho_{l} c_{l}+\rho_{r} c_{r}}, \\
& p_{I, r}=\frac{\rho_{l} c_{l} p_{r}+\rho_{r} c_{r} p_{l}+\rho_{l} c_{l} \rho_{r} c_{r}\left(u_{l}-u_{r}\right)}{\rho_{l} c_{l}+\rho_{r} c_{r}}-\frac{\sigma \kappa_{I} n \rho_{r} c_{r}}{\rho_{l} c_{l}+\rho_{r} c_{r}},
\end{aligned}
$$

and the density is calculated by performing an isentropic extrapolation from each side.

\subsection{Curvature computation}

The curvature of the interface is computed via a weighted least squares polynomial fitting method [21]. In this approach, the points of the Lagrangian mesh are used to locally fit a quartic polynomial, which is then differentiated in order to compute the local curvature. The curvature is thus known on the marker points of the Lagrangian grid and needs to be transferred to the underlying Eulerian grid. Several options may be used for this procedure, such as assigning the value of the nearest interface point or computing an arithmetic or weighted average within a certain radius. In the present work we use the weighting function of Peskin [22], as it was found to be robust and give satisfactory results.

A preliminary test was carried out in order to assess the behaviour of the method for the computation of the curvature. In this test we placed one quadrant of a circle of radius $R=1 \mathrm{~m}$, centred at $(x, y)=(0 \mathrm{~m}, 0 \mathrm{~m})$ within a computational domain of size $\boldsymbol{x}(m) \in[0,2]^{2}$. Three different grids were employed in order to demonstrate mesh convergence. The curvature field and its properties (after being transferred to the Eulerian grid) are plotted in figure 2. It is shown that curvature converges with second order accuracy for both the mean and the standard deviation. The relative error is very small; about $1 \%$ for a grid with 8 points per circle radius.

\section{Verification of the method}

The performance of the method is assessed through a series of test cases. In these problems, the effects of capillarity are amplified by considering an unrealistically large value for the surface tension coefficient $\left(\sigma=10^{5} \mathrm{~N} / \mathrm{m}\right.$, whereas $\sigma \simeq 0.072 \mathrm{~N} / \mathrm{m}$ for water-air interfaces at $25^{\circ} \mathrm{C}$ ). As a result, the flows under study are primarily (or solely) driven by surface tension. For the purpose of demonstrating the qualities of the proposed formulation, no redistribution of the Lagrangian grid takes place (unless otherwise stated). ${ }^{2}$ The implications of this are twofold: on one hand this means that all perturbations (even of infinitesimal scale) are allowed to grow freely, as they will not be artificially smoothed out (and thus might end up being catastrophic for the computations). This comes in addition to the already challenging situation created by the absence of viscosity and the low numerical dissipation of the employed high-order numerical

\footnotetext{
${ }^{2}$ Redistribution is a process that is required in cases of large deformations of the front (Lagrangian grid), with the target of maintaining a well-defined mesh with more or less equal in size elements of good aspect ratio.
} 


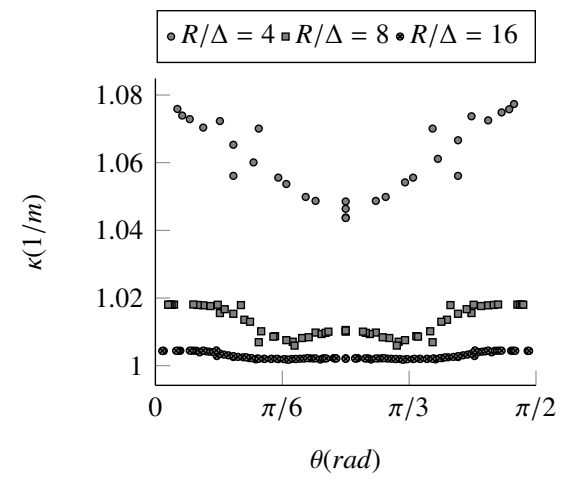

(a) Eulerian curvature field.

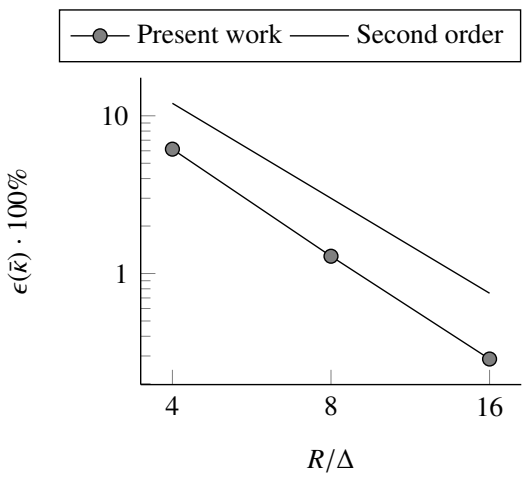

(b) Relative error in average curvature.

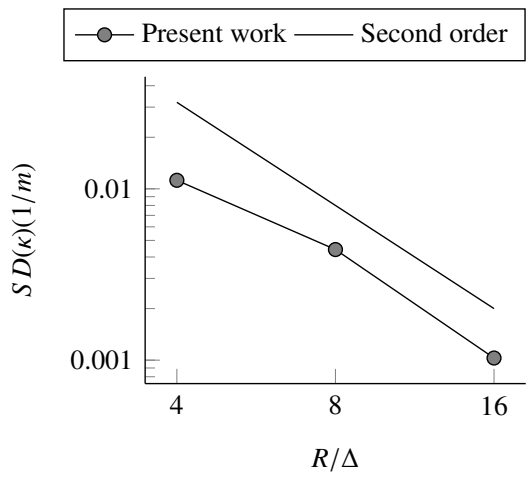

(c) Standard deviation.

Figure 2: Eulerian curvature field properties for a $R=1 \mathrm{~m}$ quarter circle.

scheme. On the other hand, redistribution of the interface could disrupt the method's ability to relax to an equilibrium by introducing perturbations in the curvature field.

\subsection{Problem 1: Static droplet with exact curvature}

The first problem to be considered was a two-dimensional water $\left(\rho_{w}=1000 \mathrm{~kg} / \mathrm{m}^{3}\right)$ droplet $(R=1 \mathrm{~m})$ in air $\left(\rho_{a}=1 \mathrm{~kg} / \mathrm{m}^{3}\right)$. The computational domain was a square of size $\boldsymbol{x}(m) \in[0,2]^{2}$ (taking into consideration the problem's symmetry about the $x$ and $y$ axes), discretized with 8 points in each direction (yielding a resolution of (maximum) 4 points per droplet radius). The aim of this test was to validate the well-balancedness property of the proposed method (i.e. consistency between the discretization of surface tension and pressure forces). For this reason, curvature was set to its exact value $\kappa=1 / R$, instead of being computed as described in section 2.2. The simulation was conducted for a physical time equal to $t_{\max }=1.5 \mathrm{~s}$, which is longer than the surface tension time scale of the problem $\left(T_{\sigma}=\sqrt{\rho D^{3} / \sigma} \simeq 0.283 s\right)$. Exact balance was maintained throughout the simulation, with no currents being generated. The method was also able to reach an equilibrium (to within round-off errors) when a perturbed initial state was defined (e.g. by setting the pressure inside the droplet equal to the external). After a transient period, the maximum error in the dimensionless velocity field was of the order $L_{\infty}\left(|\boldsymbol{u}| / c_{a, 0}\right) \approx 10^{-15}$.

\subsection{Problem 2: Static droplet with computed curvature}

The second test case was similar to the problem considered in section 3.1 with the only difference being that the curvature field was computed as described in section 2.2. These computations carry errors (as discussed in section 2.2), meaning that the resulting surface tension forces do not balance the initial (exact) pressure field. Velocity and acoustic fields are generated as a response to this imbalance. For a numerical method, the ability to progress towards a numerical equilibrium is a much desired property: the interface should be deformed in a way that the computed surface tension (i.e. the curvature field) and pressure forces cancel out. Simulations were run until $t_{\max }=1.5 \mathrm{~s}$, corresponding to $t_{\max } \simeq 5.3 T_{\sigma}$ surface tension time scales. Three different grids (with $R / \Delta=4,8$ and 16 points per droplet radius) were used in order to assess the method's behaviour with mesh refinement. Results are plotted in figure 3 .

Figure 3a shows the Eulerian curvature field at the beginning and end of the simulation (for the case where $R / \Delta=4)$. The curvature field has relaxed to a near-equilibrium, at about the mean value of the initial cosinusoidalshaped field. The standard deviation of the field dropped from about $S D /\left.\mu(k)\right|_{t=0 s} \simeq 1 \%$ of the mean to less than $S D /\left.\mu(k)\right|_{t=t_{\max }} \simeq 0.04 \%$ of it. In figure $3 \mathrm{~b}$, the pressure along the $y=x$ line is plotted, at $t=1.5 \mathrm{~s}$. The profiles are sharp and flat (no oscillations are observed). The pressure inside the droplet has increased slightly from the initially prescribed and exact value in order to balance the computed surface tension forces. The coefficient of variation (i.e. the ratio between the standard deviation and the mean) of the droplet pressure at $t=1.5 \mathrm{~s}$ is 


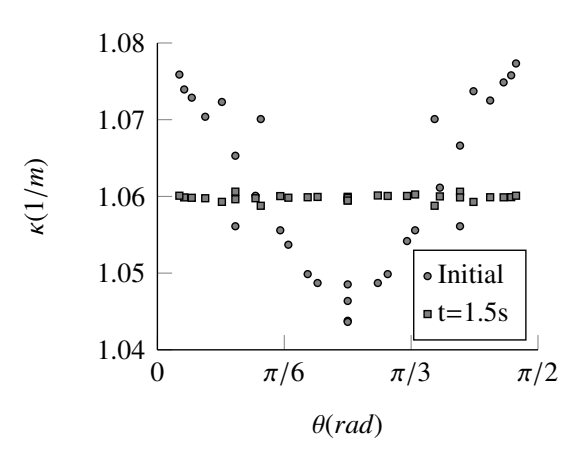

(a) Eulerian curvature field, $R / \Delta=4$.

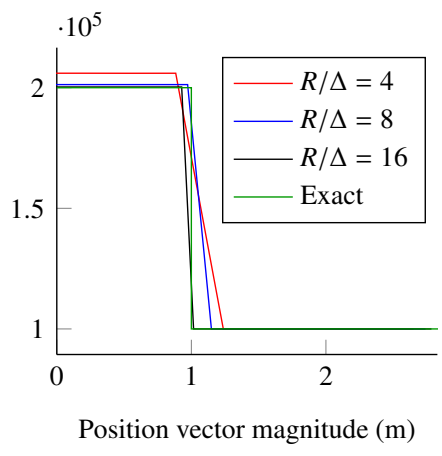

(b) Pressure along the $y=x$ line, $t=1.5 \mathrm{~s}$.

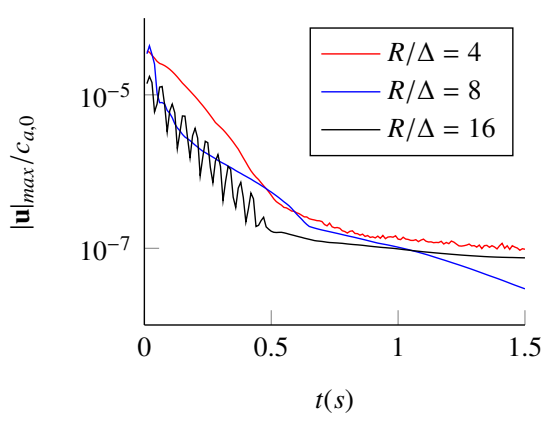

(c) Maximum velocity magnitude along time.

Figure 3: Static droplet with computed curvature.

$C V=S D /\left.\mu\left(p_{\text {droplet }}\right)\right|_{t=t_{\max }} \simeq 0.003 \%$ (for the case where $R / \Delta=4$ ), indicating that the pressure field is practically constant. Note that the interface is locally aligned to the grid at certain polar angles (rendering the normal-to-the-grid and normal-to-the-interface formulations equivalent) while at others is not; this suggests that the differences between the two are small. Lastly, the relative percentage error in the average droplet pressure for different grid resolutions reduces at second-order rate, similar to the order of curvature computations (figure $2 b$ ).

Velocity currents are typically reported in terms of the maximum capillary number $C a=|\mathbf{u}|_{\max } \mu / \sigma$, however our model does not include viscosity and this number is undefined. We instead consider the time history of the maximum dimensionless velocity magnitude. This is plotted in figure $3 \mathrm{c}$ for different grid resolutions. The magnitude of the currents is markedly small and decreases with time, suggesting that the method progressed towards an equilibrium. The above holds for all tested grids. In the test case presented in section 3.1, it was shown that if an equilibrium is reached it will be preserved. This means that following the transient period required to reach this approximate equilibrium (where the pressure field is almost constant) no further imbalance exists and currents are no longer generated. The remaining ones will eventually flow out of the domain, though at a much larger time scale.

\subsection{Problem 3: Translating droplet with computed curvature}

The test case presented in this section is similar to the problem considered in section 3.2 with an added characteristic: the droplet is simultaneously being translated by an underlying velocity field. The aim of such a test is to validate the implementation of capillary action in the presence of inertial forces. In the original translating droplet problem as proposed by Popinet [23], the droplet was advected by a uniform underlying velocity field in a direction that was parallel to the grid. This is an idealized scenario where grid-based effects are disregarded. In the current work, we introduce a new translating droplet test case with reduced grid bias. This is of particular importance for the presently proposed method, due to its grid-aligned flavour. In this test case, translation is performed at an arbitrary angle with respect to the grid. Angles with special properties (e.g. $\alpha=45^{\circ}$ ) should not be considered, so as to avoid secondary effects due to symmetry. In the translating reference frame the solution should be similar to the static droplet case.

The domain consisted of a rectangle with $x(m) \in[-4,12]$ and $y(m) \in[-4,8]$. Three different grids were used, yielding a (maximum) resolution of 4,8 and 16 points along the droplet radius. The droplet of radius $R=1 \mathrm{~m}$ was initially placed at $(x, y)=(0 m, 0 m)$. The underlying velocity field was set to $\boldsymbol{U}(\mathrm{m} / \mathrm{s})=(10,5)$, forming an angle equal to $\alpha \simeq 26.565^{\circ}$ with the grid. A new time scale related to the advection is thus introduced: $T_{a}=D /|\boldsymbol{U}| \simeq 0.179 \mathrm{~s}$. The ratio between the surface tension and advection time scales is related to the Weber number $W e=\left(T_{\sigma} / T_{a}\right)^{2}$, which describes the ratio of inertial to capillary forces. The underlying velocity field $\boldsymbol{U}$ was chosen such that inertial and surface tension forces are of similar order of magnitude: the Weber number is equal to $W e=2.5$. The simulations were run till physical time $t=0.8 \mathrm{~s}$, which is larger than both surface tension and inertial time scales. At the end of the simulation, the droplet has covered a distance of about 4.5 times its diameter. 


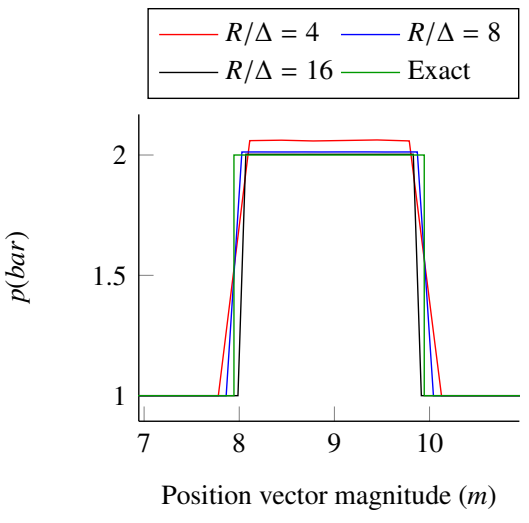

(a) Pressure along the $y=x-4$ line, $t=0.8 s$.

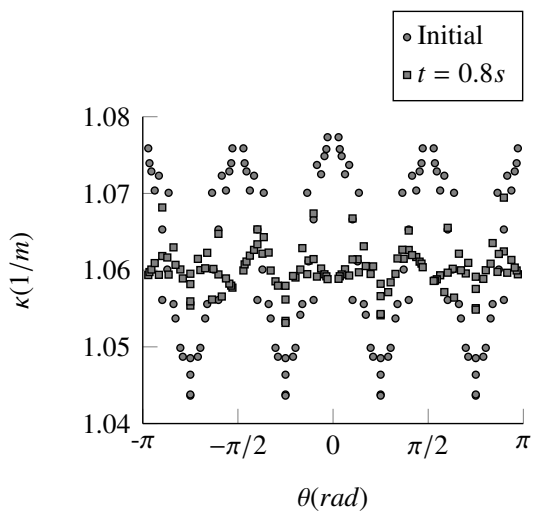

(b) Eulerian curvature field, $R / \Delta=4$.

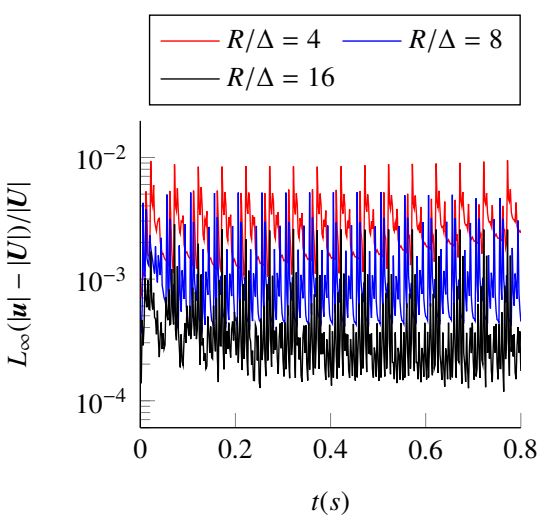

(c) Time history of velocity fluctuations.

Figure 4: Translating droplet with computed curvature.

Figure 4a shows the droplet pressure profiles at $t=0.8 \mathrm{~s}$ for different grid resolutions. The profiles are extracted along the $y=x-4$ line (which forms an $\alpha=45^{\circ}$ angle with the underlying mesh and the effective resolution is thus lower). The pressure inside the droplet is practically constant, no oscillations appear and the jump is fully sharp. The errors in the average droplet pressure are similar in magnitude with the static case, and also converge with second order rate (accuracy of curvature computations). The Eulerian curvature field is plotted in figure $4 \mathrm{~b}$ at the start $(t=0 s)$ and end $(t=0.8 s)$ of the droplet translation. The standard deviation of the curvature field is reduced, signifying a trend towards equilibrium. Finally, in figure $4 \mathrm{c}$ we plot the time history of the maximum relative error in the velocity (with the flow field being stored every $t=2 \mathrm{~ms}$ ). The magnitude of the currents is small and their mean remains roughly constant with time. Moreover, they are shown to be reducing with grid refinement. The fluctuating behaviour is related to the pure-cell definition of the Eulerian topology (i.e. to the component changes as the droplet advances), which is in turn related to the spatial discretization (the frequency reduces with mesh refinement).

\subsection{Problem 4: Oscillating drop}

The last case to be considered for the validation of the method was that of an oscillating elliptical drop. Similar configurations are often used to validate numerical methods (e.g. [5, 3]). The domain was a square of size $\boldsymbol{x}(m) \in$ $[0,2]^{2}$ (taking into consideration the problem's symmetry about the $x$ and $y$ axes). The droplet was centred at $(x, y)=$ $(0 \mathrm{~m}, 0 \mathrm{~m})$ and its major and minor semi-axes were equal to $a=5 / 4 \mathrm{~m}, b=4 / 5 \mathrm{~m}$. Three different grids were used, with 32, 64 and 128 points in each direction. The Lagrangian grid was redistributed every two hundred time steps (as this is a dynamic problem with large deformations). The period of droplet oscillation $T$ can be computed analytically (for the case of inviscid and incompressible fluids) as $T=2 \pi \sqrt{\left(\rho_{l}+\rho_{a}\right) R^{3} /(6 \sigma)}$, where $R=\sqrt{a b}$ is the equivalent circular radius of the droplet [3]. Simulations were performed till $t_{\max }=1 \mathrm{~s}$, equal to $\simeq 4$ drop oscillation periods.

The kinetic energy per unit volume (integrated over the simulated quadrant) was used to measure the numerically predicted oscillation period. Figure 5a shows the time history of the kinetic energy for different mesh resolutions; very good agreement with the analytical result for the oscillation period (depicted with dashed vertical lines) is found in all cases (the small discrepancy is attributed to the different assumptions). The observed decay is due to numerical dissipation and acoustic radiation (for a comparison with the literature see [3]). In figure $5 \mathrm{~b}$ the droplet interface is plotted at different time instants. The losses in energy are reflected in the more circular shape of the droplet as time advances. The same conclusion can be drawn from figure $5 \mathrm{c}$, which plots the droplet interface (for different grids) after completion of three oscillation cycles. In general, the method is shown to be capable of predicting dynamic phenomena due to capillary action with success. 


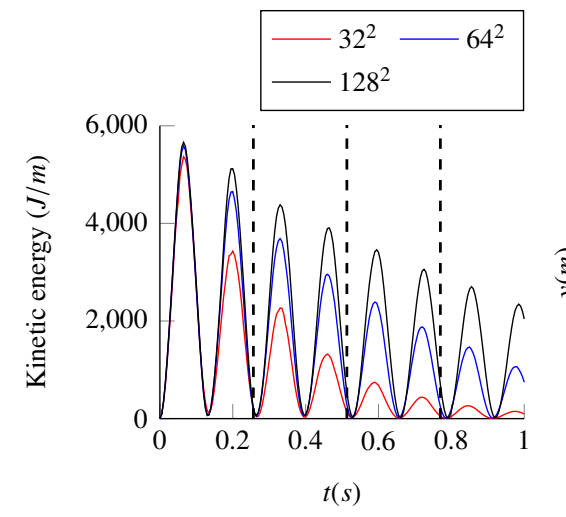

(a) Time history of the kinetic energy for differ- (b) ent grid resolutions.

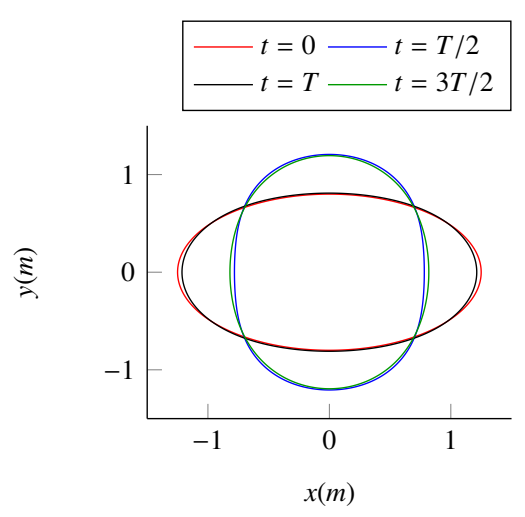
$128^{2}$.

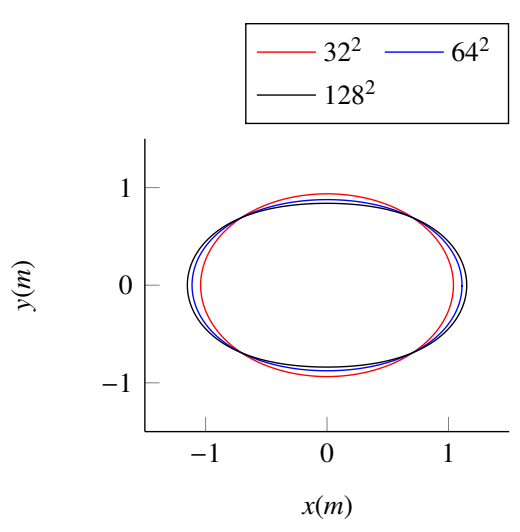

(c) Droplet interface for different grid resolutions, $t=3 T$.

Figure 5: Oscillating elliptical drop.

The oscillating drop problem was also examined in three dimensions, with the drop initially having a spheroidal shape. The simulation was performed without taking advantage of any symmetries (the computational domain was a box of size $\boldsymbol{x}(m) \in[-2,2]^{3}$, discretized with 64 points in each direction). Figure 6 shows the drop at various moments of its first oscillation cycle. An animation of this process, labelled as Video 1, is available as supplementary material.

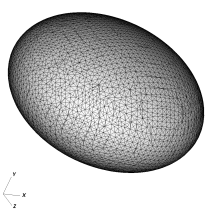

(a) $t=0 \mathrm{~s}$.

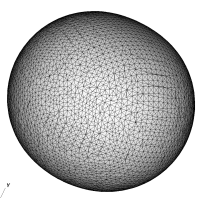

(b) $t=0.05 \mathrm{~s}$

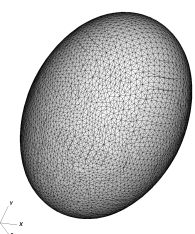

(c) $t=0.1 \mathrm{~s}$

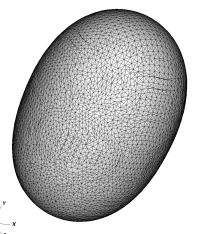

(d) $t=0.15 \mathrm{~s}$.

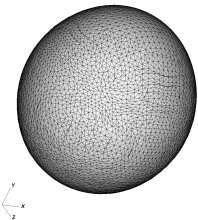

(e) $t=0.2 \mathrm{~s}$.

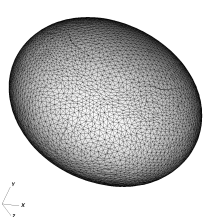

(f) $t=0.25 \mathrm{~s}$.

Figure 6: Three-dimensional simulation of the surface-tension-driven shape oscillations of an initially spheroidal drop.

\section{Shock-induced droplet breakup}

The developed method was then applied to the study of a problem where compressibility and capillarity are both important: the shock-driven breakup of a cylindrical water droplet in air. The set-up replicates the experiments of Igra and Takayama [24] which have been used by several researchers as a benchmark for the validation of their numerical methods $[6,2,3,11]$. In the present work, the problem of shock-induced droplet breakup was revisited, with the aim of investigating the effects of surface tension (which were not considered in our previous work [11]). A cylindrical water droplet of diameter $d=0.0048 \mathrm{~m}$ was placed in a domain of size $x \in[-0.036 \mathrm{~m}, 0.036 \mathrm{~m}], y \in[0 \mathrm{~m}, 0.036 \mathrm{~m}]$, in equilibrium with the surrounding air. ${ }^{3}$ Only one half of the droplet was simulated, due to symmetry (about the $x$ axis). The droplet was centred at $(x, y)=(0 \mathrm{~m}, 0 \mathrm{~m})$. The density of water was set to $\rho_{w}=1000 \mathrm{~kg} / \mathrm{m}^{3}$ and that of

\footnotetext{
${ }^{3}$ The effects of surface tension are not realistic; a three-dimensional simulation would be required for such an investigation (i.e. to compare with available experiments). The herein presented 2D simulation was however deemed sufficient for demonstrating the capabilities and potential of the method, which is the purpose of this work.
} 
air at $\rho_{a}=1.2 \mathrm{~kg} / \mathrm{m}^{3}$. Both fluids were initially at rest under atmospheric pressure conditions. The surface tension coefficient was set to $\sigma=0.073 \mathrm{~N} / \mathrm{m}$. Post-shock conditions (corresponding to a $M=1.47$ shock wave in air) were defined at the left boundary, while non-reflecting conditions were set at the two remaining boundaries. The domain was discretized with a resolution corresponding to 50 points per initial droplet radius, which was shown to be sufficient for an accurate prediction of the breakup dynamics [11]. Redistribution of the Lagrangian grid was carried out every twenty time steps.

Figure 7 shows the effects of surface tension on the predicted interface at four different time instants following shock impact. The role of surface tension is negligible during the initial (earlier than depicted) stages of the breakup process. This may also be verified by considering the Weber number, $W e=\rho_{a} u_{a}^{2} D / \sigma \approx O\left(10^{3}\right)$. However, differences are observed following the formation of tips on the top of the cylinder (i.e. at the onset of the stripping process). This process is not instantaneous; the cylinder remains a coherent body for a considerable amount of time thereafter. During this stage (and also following the detachment of filaments) surface tension has a considerable influence on the flow. This is also reflected in figure 8, where the flow is depicted through numerical Schlieren contours; the vortex shedding and wake patterns are different. A non-negligible delay in the breakup is also noted: in the simulations without surface tension, the first pinch-off event is observed at $t=561 \mathrm{~ns}$ following shock impact, whereas if surface tension is considered the same filament is cut off at $t=601 n s$, i.e. with a delay of about $\simeq 7 \%$.

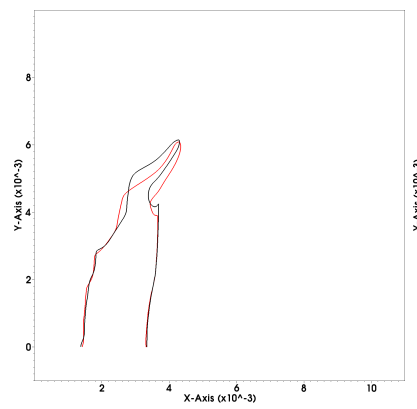

(a) $t=400 \mu \mathrm{s}$.

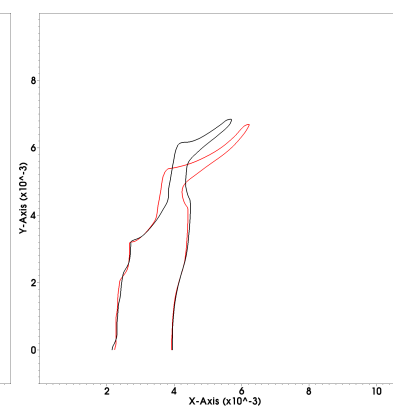

(b) $t=450 \mu \mathrm{s}$

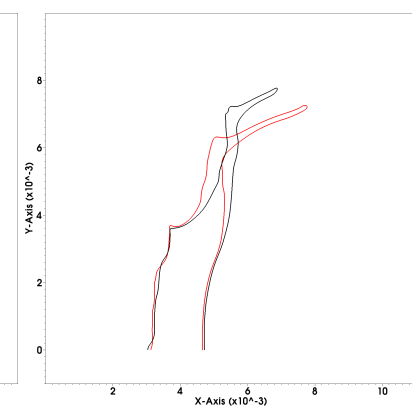

(c) $t=500 \mu \mathrm{s}$.

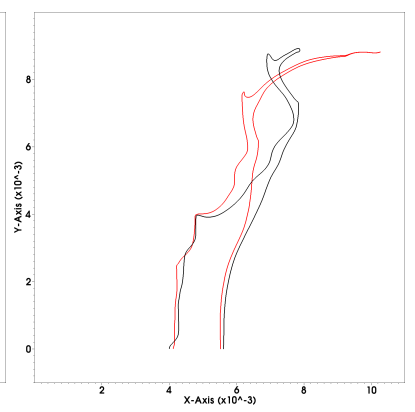

(d) $t=550 \mu \mathrm{s}$.

Figure 7: Droplet interface at various time instants following shock impact for simulations with (red) and without (black) surface tension, 2D simulation of the high-speed breakup of a water column in air.

\section{Conclusions}

A novel way for incorporating surface tension forces in sharp-interface compressible flow models was proposed. The method is designed in adherence to the concepts of the grid-aligned formulation for ghost fluid methods, and maintains the simplicity and benefits of the original formulation to a great extent. Validation through several challenging test cases showed that the method produces highly accurate results, and is capable of predicting both static and dynamic phenomena due to capillary action with success.

We note that the proposed framework is general, as any ghost fluid method can be formulated along the herein presented lines. Moreover, it may be used in conjunction with any sharp-interface technique, such as a level set method. However, further validation of such a combination is essential, due to the lower accuracy of level sets compared to front tracking and the differences in the computations of the curvature.

\section{Acknowledgements}

This work was carried out in the framework of the HAoS project, which has received funding from the European Union Horizon 2020 Research and Innovation programme, grant agreement no. 675676. The authors would like to thank Professors J. Glimm and X. Li of Stony Brook University for the use of their front tracking framework. 


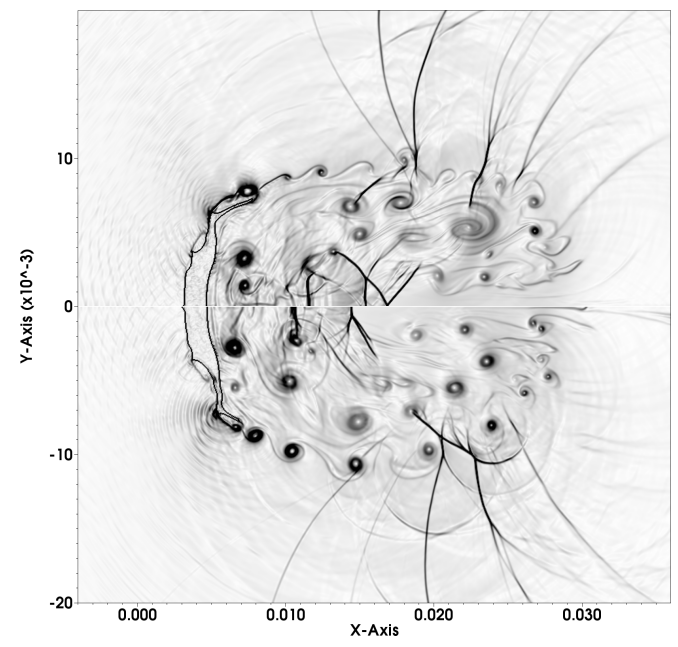

(a) $t=500 \mu \mathrm{s}$

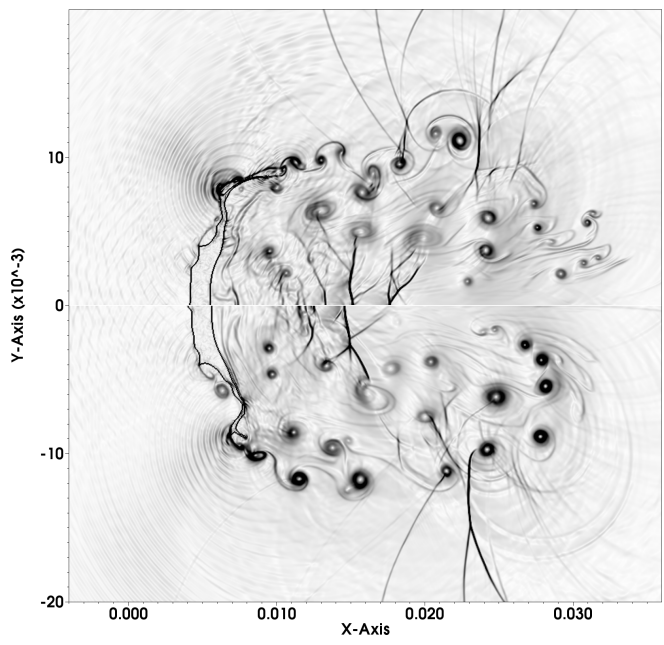

(b) $t=550 \mu \mathrm{s}$.

Figure 8: Numerical Schlieren contour plots (magnitude of density gradient) at two time instants following shock impact for simulations with (upper half) and without (lower half) surface tension, 2D simulation of the high-speed breakup of a water column in air.

\section{References}

[1] S. Popinet, Numerical models of surface tension, Annual Review of Fluid Mechanics 50 (2018) 49-75.

[2] K. Schmidmayer, F. Petitpas, E. Daniel, N. Favrie, S. Gavrilyuk, A model and numerical method for compressible flows with capillary effects, Journal of Computational Physics 334 (2017) 468-496.

[3] D. P. Garrick, M. Owkes, J. D. Regele, A finite-volume HLLC-based scheme for compressible interfacial flows with surface tension, Journal of Computational Physics 339 (2017) 46-67.

[4] Z. Jibben, J. Velechovsky, T. Masser, M. M. Francois, Modeling surface tension in compressible flow on an adaptively refined mesh, Computers \& Mathematics with Applications 78 (2019) 504-516.

[5] G. Perigaud, R. Saurel, A compressible flow model with capillary effects, Journal of Computational Physics 209 (2005) 139-178.

[6] H. Terashima, G. Tryggvason, A front-tracking/ghost-fluid method for fluid interfaces in compressible flows, Journal of Computational Physics 228 (2009) 4012-4037.

[7] B. Braconnier, B. Nkonga, An all-speed relaxation scheme for interface flows with surface tension, Journal of Computational Physics 228 (2009) 5722-5739.

[8] W. Bo, X. Liu, J. Glimm, X. Li, A robust front tracking method: Verification and application to simulation of the primary breakup of a liquid jet, SIAM Journal on Scientific Computing 33 (2011) 1505-1524.

[9] C.-H. Chang, X. Deng, T. G. Theofanous, Direct numerical simulation of interfacial instabilities: a consistent, conservative, all-speed, sharp-interface method, Journal of Computational Physics 242 (2013) 946-990.

[10] D. Fuster, S. Popinet, An all-Mach method for the simulation of bubble dynamics problems in the presence of surface tension, Journal of Computational Physics 374 (2018) 752-768.

[11] N. Bempedelis, Y. Ventikos, A simplified approach for simulations of multidimensional compressible multicomponent flows: The grid-aligned ghost fluid method, Journal of Computational Physics 405 (2020) 109129.

[12] J. Glimm, E. Isaacson, D. Marchesin, O. McBryan, Front tracking for hyperbolic systems, Advances in Applied Mathematics 2 (1981) 91-119.

[13] J. Glimm, O. A. McBryan, A computational model for interfaces, Advances in Applied Mathematics 6 (1985) 422-435. 
[14] J. Glimm, J. W. Grove, X. L. Li, K.-M. Shyue, Y. Zeng, Q. Zhang, Three-dimensional front tracking, SIAM Journal on Scientific Computing 19 (1998) 703-727.

[15] J. Du, B. Fix, J. Glimm, X. Jia, X. Li, Y. Li, L. Wu, A simple package for front tracking, Journal of Computational Physics 213 (2006) 613-628.

[16] R. P. Fedkiw, T. Aslam, B. Merriman, S. Osher, A non-oscillatory Eulerian approach to interfaces in multimaterial flows (the ghost fluid method), Journal of Computational Physics 152 (1999) 457-492.

[17] X. Y. Hu, B. C. Khoo, An interface interaction method for compressible multifluids, Journal of Computational Physics 198 (2004) 35-64.

[18] X. Hu, N. A. Adams, G. Iaccarino, On the HLLC Riemann solver for interface interaction in compressible multi-fluid flow, Journal of Computational Physics 228 (2009) 6572-6589.

[19] C. Farhat, J.-F. Gerbeau, A. Rallu, FIVER: A finite volume method based on exact two-phase Riemann problems and sparse grids for multi-material flows with large density jumps, Journal of Computational Physics 231 (2012) 6360-6379.

[20] A. Main, X. Zeng, P. Avery, C. Farhat, An enhanced FIVER method for multi-material flow problems with second-order convergence rate, Journal of Computational Physics 329 (2017) 141-172.

[21] X. Jiao, H. Zha, Consistent computation of first- and second-order differential quantities for surface meshes, Proceedings of the 2008 ACM Symposium on Solid and Physical Modeling (2008) 159-170.

[22] C. S. Peskin, Numerical analysis of blood flow in the heart, Journal of Computational Physics 25 (1977) 220252.

[23] S. Popinet, An accurate adaptive solver for surface-tension-driven interfacial flows, Journal of Computational Physics 228 (2009) 5838-5866.

[24] D. Igra, K. Takayama, Investigation of aerodynamic breakup of a cylindrical water droplet, Atomization and Sprays 11 (2001). 